\title{
Connecting orbits for Newtonian-like $N$-body problems
}

\author{
Kaili Xiang ${ }^{1}$, Fengying $\mathrm{Li}^{1 *}$ and Xiang $\mathrm{Yu}^{2}$
}

\section{*Correspondence:} lify0308@163.com

'School of Economics and Mathematics, Southwestern University of Finance and Economics, Chengdu, 61130, China Full list of author information is available at the end of the article

\begin{abstract}
Using variational minimizing methods, we prove the existence of a connecting orbit between the center of mass and infinity of Newtonian-like $\mathrm{N}$-body problems with Newtonian-type weak force potentials.
\end{abstract}

MSC: $34 \mathrm{~A} 34 ; 34 \mathrm{C} 25 ; 34 \mathrm{C} 37 ; 35 \mathrm{~A} 15$

Keywords: $\mathrm{N}$-body problems; variational minimizers; connecting orbits

\section{Introduction}

In the 1989 paper of Rabinowitz [1], we find the first substantial use of variational methods to study heteroclinic orbits for Hamiltonian systems. The perspective of that work appears influential for a number of papers by several authors which followed [2-15]. Especially, we would like to draw attention to Souissi [13], Maderna and Venturelli [14] and Zhang [15] for a study of the parabolic orbits for restricted 3-body problems and complete $N$ body problems. From those studies, we draw motivation for the present work: namely, we extend the results and methods of Souissi [13] and Zhang [15] to Newtonian-like $N$-body problems.

Given masses $m_{1}, \ldots, m_{N}>0$ of $N$ bodies, we study the following system of equations with Newtonian-type weak force potentials:

$$
m_{i} \ddot{q}_{i}(t)+\frac{\partial U(q)}{\partial q_{i}}=0
$$

where $q_{i} \in R^{k}, q=\left(q_{1}, \ldots, q_{N}\right), 0<\alpha<2$, and

$$
U(q)=\sum_{1 \leq i<j \leq N} \frac{m_{i} m_{j}}{\left|q_{i}-q_{j}\right|^{\alpha}} .
$$

We apply the variational minimizing method to prove the following.

Theorem 1.1 For (1.1), there exists one connecting orbit $\tilde{q}(t)=\left(\tilde{q}_{1}(t), \ldots, \tilde{q}_{N}(t)\right)$ between the center of mass and infinity such that:

(i) For any $1 \leq i \neq j \leq N$,

$$
\max _{0 \leq t \leq+\infty}\left|\tilde{q}_{i}(t)-\tilde{q}_{j}(t)\right|=+\infty
$$

(C) 2015 Xiang et al. This article is distributed under the terms of the Creative Commons Attribution 4.0 International License (http://creativecommons.org/licenses/by/4.0/), which permits unrestricted use, distribution, and reproduction in any medium, provided you give appropriate credit to the original author(s) and the source, provide a link to the Creative Commons license, and indicate if changes were made. 
(ii)

$$
\min _{0 \leq t \leq+\infty} \sum_{1}^{N} m_{i}\left|\dot{\tilde{q}}_{i}(t)\right|^{2}=2 E \geq 0 .
$$

\section{Variational minimizing critical points}

In order to find a connecting orbit of (1.1), we shall first find a solution of the system (1.1) on the open interval $(0, \tau)$ and then consider the limit orbit as $\tau \rightarrow+\infty$. To find a solution on $(0, \tau)$, we define the functional

$$
f(q)=\int_{0}^{\tau}\left(\frac{1}{2} \sum_{i=1}^{N} m_{i}\left|\dot{q}_{i}(t)\right|^{2}+U(q)\right) d t
$$

where

$$
q_{i} \in H_{\tau}=\left\{x, \dot{x} \in L^{2}[0, \tau] \mid x_{i}(0)=0, x_{i}(\tau)=a_{i}\right\}
$$

where $\left(a_{1}, \ldots, a_{i}, \ldots, a_{N}\right)$ is a central configuration for the $N$-body problems which satisfies $a_{j} \neq a_{i}, 1 \leq j \neq i \leq N$, and there is $\lambda \in R$ such that

$$
\sum_{j \neq i} \frac{m_{j} m_{i}\left(a_{j}-a_{i}\right)}{\left|a_{j}-a_{i}\right|^{\alpha+2}}=\lambda m_{i} a_{i}
$$

Since $\forall q_{i} \in H_{\tau}, q_{i}(0)=0$, for $q=\left(q_{1}, \ldots, q_{N}\right) \in H_{\tau} \times \cdots \times H_{\tau}$ we have the equivalent norm

$$
\|q\|_{\tau}=\left(\sum_{i=1}^{N} m_{i} \int_{0}^{\tau}\left|\dot{q}_{i}(t)\right|^{2} d t\right)^{1 / 2}
$$

Lemma 2.1 (Tonelli [16]) Let $X$ be a reflexive Banach space and $f: X \rightarrow R \cup\{+\infty\}$. Iff does not always take $+\infty$ and is weakly lower semi-continuous and coercive $(f(x) \rightarrow+\infty$, as $\|x\| \rightarrow+\infty)$, then $f$ attains its infimum on $X$.

Lemma 2.2 The functional $f(q)$ defined in (2.1) is weakly lower semi-continuous (w.l.s.c.) on $H_{\tau} \times \cdots \times H_{\tau}$.

Proof (1) It is well known that the norm and its square are w.l.s.c.

(2) $\forall\left\{q_{i}^{n}\right\} \subset H_{\tau}$, if $q_{i}^{n} \rightarrow q_{i}$ weakly, then by the compact embedding theorem, we have the following uniform convergence:

$$
\max _{0 \leq t \leq \tau}\left|q_{i}^{n}(t)-q_{i}(t)\right| \rightarrow 0, \quad n \rightarrow+\infty
$$

Let $S=\left\{\tilde{t} \in[0, \tau]: \exists 1 \leq i_{0} \neq j_{0} \leq N\right.$ s.t. $\left.q_{i_{0}}\left(t_{0}\right)=q_{j_{0}}\left(t_{0}\right)\right\}$ and let $m(S)$ denote the Lebesgue measure of $S$.

(i) If $m(S)=0$, then $U\left(q^{n}(t)\right) \stackrel{\text { a.e. }}{\rightarrow} U(q(t))$. From Fatou's lemma we have

$$
\int_{0}^{\tau} U(q) d t \leq \lim _{n \rightarrow \infty} \int_{0}^{\tau} U\left(q^{n}(t)\right) d t .
$$

(ii) If $m(S)>0$, then $\int_{0}^{\tau} U(q) d t=+\infty$ and $f(q)=+\infty$. 
Since $q^{n}(t) \rightarrow q(t)$ uniformly we have $\int_{0}^{\tau} U\left(q^{n}(t)\right) d t \rightarrow+\infty$, and so

$$
\varliminf_{n \rightarrow \infty} f\left(q^{n}\right) \geq f(q)
$$

The proof of the next lemma is straightforward.

Lemma $2.3 f$ is coercive on $H_{\tau} \times \cdots \times H_{\tau}$.

\section{Lemma 2.4}

(1) $f(q)$ attains its infimum on $H_{\tau} \times \cdots \times H_{\tau}$, and the minimizer $\tilde{q}^{\tau}(t)=\left(\tilde{q}_{1}^{\tau}(t), \ldots, \tilde{q}_{N}^{\tau}(t)\right)$ is a generalized solution [16].

(2) Furthermore, when $\tau \rightarrow+\infty$ and $\tilde{q}_{i}^{\tau}(t) \rightarrow \tilde{q}_{i}(t), \tilde{q}_{i}(t)$ has the following properties:

(i) for any $1 \leq i \neq j \leq N$,

$$
\max _{0 \leq t \leq+\infty}\left|\tilde{q}_{i}(t)-\tilde{q}_{j}(t)\right|=+\infty
$$

(ii)

$$
\min _{0 \leq t \leq+\infty} \sum_{1}^{N} m_{i}\left|\dot{\tilde{q}}_{i}(t)\right|^{2}=2 E .
$$

Definition 2.5 Concerning the velocities of the solution of (1.1),

$\left(1^{\circ}\right)$ if, for all $i$,

$$
\left|\dot{\tilde{q}}_{i}(t)\right| \rightarrow 0, \quad t \rightarrow+\infty
$$

we say $\tilde{q}(t)$ is a parabolic solution;

$\left(2^{\circ}\right)$ if, for all $i$,

$$
\left|\dot{\tilde{q}}_{i}(t)\right| \rightarrow v_{i}>0, \quad t \rightarrow+\infty
$$

we say $\tilde{q}(t)$ is a hyperbolic solution;

otherwise, we call it a mixed type solution.

The proof of (1) in Lemma 2.4 is obvious using Lemmas 2.1-2.3.

In the following, we will give the proofs of (2.8) and (2.9) of Lemma 2.4.

Lemma 2.6 There exist constants $c>0$ and $0<\theta<1$ independent of $\tau$ such that

$$
f\left(\tilde{q}^{\tau}\right) \leq c \tau^{\theta}
$$

Proof We choose a special orbit defined by

$$
q_{i}(t)=a_{i} t^{\beta}, \quad t \in[0, \tau], a_{i} \in R^{k},
$$


where $\left(a_{1}, a_{2}, \ldots, a_{N}\right)$ can be a given central configuration, $\frac{1}{2}<\beta<\min \left\{1, \frac{1}{\alpha}\right\}$, then

$$
\begin{aligned}
f(q(t))= & \frac{1}{2} \sum_{i=1}^{N} m_{i}\left|a_{i}\right|^{2} \int_{0}^{\tau} \beta^{2} t^{2(\beta-1)} d t+\int_{0}^{\tau} \sum_{1 \leq i<j \leq N} \frac{m_{i} m_{j}}{\left|a_{i}-a_{j}\right|^{\alpha}} t^{-\alpha \beta} d t \\
\leq & \frac{1}{2}\left(\sum_{i=1}^{N} m_{i}\left|a_{i}\right|^{2}\right) \frac{\beta^{2}}{2 \beta-1} \tau^{2 \beta-1} \\
& +\left(\sum_{1 \leq i<j \leq N} \frac{m_{i} m_{j}}{\left|a_{i}-a_{j}\right|^{\alpha}}\right) \frac{1}{1-\alpha \beta} \tau^{1-\alpha \beta} \\
\leq & c \tau^{\theta}
\end{aligned}
$$

where

$$
\theta=\max (2 \beta-1,1-\alpha \beta)
$$

and

$$
c=\frac{1}{2} \sum_{1}^{N} m_{i}\left|a_{i}\right|^{2} \frac{\beta^{2}}{2 \beta-1}+\sum_{1 \leq i<j \leq N} \frac{m_{i} m_{j}}{\left|a_{i}-a_{j}\right|^{\alpha}} \frac{1}{1-\alpha \beta}>0 .
$$

When $0<\alpha<2$, we have $\frac{1}{\alpha}>\frac{1}{2}$. We can choose $\frac{1}{2}<\beta<\frac{1}{\alpha}$, then $2 \beta-1>0,1-\alpha \beta>0$, and hence $\theta>0$. When $\beta<1,2 \beta-1<1$, then $0<\theta<1$.

Lemma 2.7 Let $\tilde{q}^{n}(t)=\left(\tilde{q}_{1}^{n}(t), \ldots, \tilde{q}_{N}^{n}(t)\right)$ be critical points corresponding to the minimizing critical values $\min _{H_{n}} f(q)$, where $H_{n}$ was defined in (2.2) when $\tau=n$. Then the maximum distance between $\tilde{q}_{i}^{n}$ and $\tilde{q}_{j}^{n}$ on $R^{+}$satisfies

$$
\left\|\tilde{q}_{i}^{n}(t)-\tilde{q}_{j}^{n}(t)\right\|_{\infty} \rightarrow+\infty, \quad \text { when } n \rightarrow+\infty
$$

Proof By the definition of $f\left(\tilde{q}^{n}\right)$ and Lemma 2.6, we have the inequalities

$$
c n^{\theta} \geq f\left(\tilde{q}^{n}\right) \geq \int_{0}^{n} \sum_{1 \leq i<j \leq N} \frac{m_{i} m_{j}}{\left|\tilde{q}_{i}^{n}(t)-\tilde{q}_{j}^{n}(t)\right|^{\alpha}} d t .
$$

Hence

$$
\sum_{1 \leq i<j \leq N} \frac{m_{i} m_{j}}{\left\|\tilde{q}_{i}^{n}(t)-\tilde{q}_{j}^{n}(t)\right\|_{\infty}^{\alpha}} \leq c n^{\theta-1} \rightarrow 0
$$

from which it follows that $\forall 1 \leq i<j \leq N,\left\|\tilde{q}_{i}^{n}(t)-\tilde{q}_{j}^{n}(t)\right\|_{\infty} \rightarrow+\infty, n \rightarrow+\infty$.

Lemma 2.8 $\left\{\tilde{q}^{n}(t)\right\}$ is equi-continuous and uniformly bounded on any compact interval.

Proof By the proof of Lemma 2.6, we can see $\forall T>0$,

$$
\sum_{i=1}^{N} m_{i} \int_{0}^{T}\left|\dot{\tilde{q}}_{i}^{n}(t)\right|^{2} d t \leq c T^{\theta}
$$


Then, for any $0 \leq s, r \leq T$, we have

$$
\begin{aligned}
\left|\tilde{q}_{i}^{n}(s)-\tilde{q}_{i}^{n}(r)\right| & \leq \int_{r}^{s}\left|\dot{\tilde{q}}_{i}^{n}(t)\right| d t \\
& \leq|s-r|^{1 / 2}\left(\int_{r}^{s}\left|\dot{\tilde{q}}_{i}^{n}(t)\right|^{2} d t\right)^{1 / 2} \\
& \leq\left(\frac{c T^{\theta}}{m_{i}}\right)^{1 / 2}|s-r|^{1 / 2} .
\end{aligned}
$$

By $q^{n}(0)=0$ and the above inequality, for $0<s<T$, we have

$$
\left|\tilde{q}_{i}^{n}(s)\right| \leq\left(\frac{c T^{\theta}}{m_{i}}\right)^{1 / 2}|s|^{1 / 2} \leq\left(\frac{c T^{\theta}}{m_{i}}\right)^{1 / 2} T^{1 / 2} .
$$

Now we can prove Theorem 1.1.

Proof of Theorem 1.1 For any compact interval $[a, b]$ of $R^{+}$, Marchal's theorem [17] implies that $\tilde{q}^{n}(t)$ has no collision on $(a, b)$, so, by the Ascoli-Arzelà theorem, we know $\left\{\tilde{q}^{n}\right\}$ has a sub-sequence converging uniformly to a limit $\tilde{q}(t)$ on any compact set $[c, d] \subset(a, b)$, and $\tilde{q}(t) \in C^{2}\left(R^{+}, R^{k}\right)$ is a solution of (1.1). By the energy conservation law and (2.17), we have

$$
E=\sum_{i=1}^{N} \frac{1}{2} m_{i}\left|\dot{\tilde{q}}_{i}\right|^{2}-\sum_{1 \leq i<j \leq N} \frac{m_{i} m_{j}}{\left|\tilde{q}_{i}-\tilde{q}_{j}\right|^{\alpha}} \geq 0
$$

rewritten as

$$
\sum_{i=1}^{N} \frac{1}{2} m_{i}\left|\dot{\tilde{q}}_{i}\right|^{2}=\sum_{1 \leq i<j \leq N} \frac{m_{i} m_{j}}{\left|\tilde{q}_{i}-\tilde{q}_{j}\right|^{\alpha}}+E
$$

Now we claim:

(i) for any $1 \leq i \neq j \leq N$,

$$
\max _{t \in R^{+}}\left|\tilde{q}_{i}(t)-\tilde{q}_{j}(t)\right|=+\infty
$$

suppose there exist $1 \leq i_{0}<j_{0} \leq N$ and $d>0$ such that

$$
\left|\tilde{q}_{i_{0}}(t)-\tilde{q}_{j_{0}}(t)\right|<d, \quad \forall t \in R^{+} .
$$

By (2.24), there exist $1 \leq k_{0} \leq N$ and $e>0$ such that

$$
\left|\dot{\tilde{q}}_{k_{0}}\right|>e, \quad \forall t \in R^{+}
$$

then we have

$$
c t^{\theta} \geq \frac{1}{2} \int_{0}^{t} \sum_{i=1}^{N} m_{i}\left|\dot{\tilde{q}}_{i}\right|^{2} d t \geq \frac{1}{2} \int_{0}^{t} m_{k_{0}}\left|\dot{\tilde{q}}_{k_{0}}\right|^{2} d t \geq \frac{1}{2} m_{k_{0}} e^{2} t .
$$

This is a contradiction, since $0<\theta<1$ and $t \in R^{+}$. 
Now by (2.24), we have:

(ii) $\min _{t \in R^{+}} \sum_{i=1}^{N} m_{i}\left|\dot{\tilde{q}}_{i}(t)\right|^{2}=2 E \geq 0$

\section{Competing interests}

The authors declare that they have no competing interests.

\section{Authors' contributions}

The research and writing of this manuscript was a collaborative effort from all the authors. All authors read and approved the final manuscript.

\section{Author details}

${ }^{1}$ School of Economics and Mathematics, Southwestern University of Finance and Economics, Chengdu, 61130, China. ${ }^{2}$ Department of Mathematics, Sichuan University, Chengdu, 610064, China.

\section{Acknowledgements}

The authors sincerely thank the referees for their many valuable comments which help us improving the paper. This paper was partially supported by NSF of China (No. 11071175 and No. 11426181) and Fundamental Research Funds for the Central Universities (JBK 130401 and JBK 150931).

\section{Received: 16 November 2014 Accepted: 26 May 2015 Published online: 16 June 2015}

\section{References}

1. Rabinowitz, PH: Periodic and heteroclinic orbits for a periodic Hamiltonian system. Ann. Inst. Henri Poincaré, Anal. Non Linéaire 6(5), 331-346 (1989)

2. Caldiroli, P, Jeanjean, L: Homoclinics and heteroclinics for a class of conservative singular Hamiltonian systems. J. Differ. Equ. 136(1), 76-114 (1997)

3. Chen, C-N, Tzeng, S-Y: Periodic solutions and their connecting orbits of Hamiltonian systems. J. Differ. Equ. 177(1), 121-145 (2001)

4. Felmer, PL: Heteroclinic orbits for spatially periodic Hamiltonian systems. Ann. Inst. Henri Poincaré, Anal. Non Linéaire 8(5), 477-497 (1991)

5. Mather, JN: Variational construction of connecting orbits. Ann. Inst. Fourier (Grenoble) 4, 1349-1386 (1993)

6. Maxwell, TO: Heteroclinic chains for a reversible Hamiltonian system. Nonlinear Anal., Theory Methods Appl. 28, 871-887 (1997)

7. Rabinowitz, PH: A variational approach to heteroclinic orbits for a class of Hamiltonian systems. In: Dautry, R (ed.) Frontiers in Pure and Applied Mathematics, pp. 267-278. North-Holland, Amsterdam (1991)

8. Rabinowitz, PH: Heteroclinics for a reversible Hamiltonian system II. Differ. Integral Equ. 7(5-6), 1557-1572 (1994)

9. Rabinowitz, PH: Heteroclinics for a reversible Hamiltonian system. Ergod. Theory Dyn. Syst. 14(4), $817-829$ (1994)

10. Rabinowitz, PH: Connecting orbits for a reversible Hamiltonian system. Ergod. Theory Dyn. Syst. 20(6), 1767-1784 (2000)

11. Rabinowitz, PH: A note on a class of reversible Hamiltonian systems. Adv. Nonlinear Stud. 9(4), 815-823 (2009)

12. Rabinowitz, PH, Tanaka, K: Some results on connecting orbits for a class of Hamiltonian systems. Math. Z. 206(3), 473-499 (1991)

13. Souissi, C: Existence of parabolic orbits for the restricted three-body problem. An. Univ. Craiova, Ser. Mat. Inform. 31, 85-93 (2004)

14. Maderna, E, Venturelli, A: Globally minimizing parabolic motions in the Newtonian N-body problem. Arch. Ration. Mech. Anal. 194, 283-313 (2009)

15. Zhang, S: Variational minimizing parabolic and hyperbolic orbits for the restricted 3-body problems. Sci. China Math. $55,721-725(2012)$

16. Tonelli, L: Sull'integrazione per parti. Atti Accad. Naz. Lincei (5) 18(2), 246-253 (1909)

17. Marchal, C: How the method of minimization of action avoids singularities. Celest. Mech. 83, 325-353 (2002) 